\title{
The BDX experiment at Jefferson Laboratory
}

\author{
Andrea Celentano ${ }^{1, a}$, for the BDX collaboration \\ ${ }^{1}$ INFN-Genova, via Dodecaneso 33, 16146 Genova, Italy
}

\begin{abstract}
The existence of MeV-GeV dark matter (DM) is theoretically well motivated but remarkably unexplored. The Beam Dump eXperiment (BDX) at Jefferson Laboratory aims to investigate this mass range. Dark matter particles will be detected trough scattering on a segmented, plastic scintillator detector placed downstream of the beam-dump at one of the high intensity JLab experimental Halls. The experiment will collect up to $10^{22}$ electrons-on-target (EOT) in a one-year period. For these conditions, BDX is sensitive to the DM-nucleon elastic scattering at the level of a thousand counts per year, and is only limited by cosmogenic backgrounds. The experiment is also sensitive to DM-electron elastic and inelastic scattering, at the level of 10 counts/year. The foreseen signal for these channels is an high-energy $(>100 \mathrm{MeV})$ electromagnetic shower, with almost no background. The experiment, has been presented in form of a Letter of Intent to the laboratory, receiving positive feedback, and is currently being designed.
\end{abstract}

\section{Introduction: direct Dark Matter searches in beam dump experiments}

\subsection{Physics motivations}

Although overwhelming astrophysical and cosmological evidence supports the existence of DM, its elementary properties remain largely elusive. The expectation that dark matter has some kind of interaction with Standard Model matter is strongly motivated by two possible mechanisms to explain its origin - either as an annihilation product of thermal Standard Model matter in the early Universe, or as a product of the same unknown processes that generate the baryon asymmetry. There is currently an active program to probe particle DM scattering with direct detection experiments, annihilation with indirect detection telescopes, and production with particle accelerators. However, most of these efforts are designed to search for heavy (10-1000 GeV) DM candidates and sharply lose sensitivity to lighter (sub-GeV) states whose signals are either too feeble to detect or lie in high-background regions.

In fact, dark matter candidates are readily motivated in the entire $\mathrm{MeV}$-to-TeV range. Much heavier dark matter is disfavored because its naive thermal abundance exceeds the observed cosmological matter density. Much beneath an $\mathrm{MeV}$, astrophysical and cosmological constraints allow only dark matter with ultra-weak couplings to quarks and leptons [1]. Between these boundaries (MeV - TeV), simple models of dark matter can account for its observed abundance through either thermal freeze-out or non-thermal mechanisms. The importance of broadening the experimental search program to include the $\mathrm{MeV}-\mathrm{GeV}$ mass range is underscored by the lack of evidence for

a e-mail: andrea.celentano@ge.infn.it weak-scale $(\gtrsim 100 \mathrm{GeV})$ dark matter scattering through $Z$ bosons, ever stronger constraints on Higgs mediated scattering, and by the absence to date of evidence for new SMcharged matter at the LHC.

The status of experimental searches for $\mathrm{MeV}-\mathrm{TeV}$ dark matter is summarized in the following. The best constraints on multi-GeV dark matter interactions are from underground searches for nuclei recoiling off nonrelativistic dark matter particles in the Galactic halo. However, these searches are insensitive to few-GeV or lighter dark matter, whose nuclear scattering transfers invisibly small kinetic energy to a recoiling nucleus. Direct detection using electron-scattering offers an alternative strategy to search for sub-GeV dark matter, but with dramatically higher backgrounds [2-4]. Among the bestmotivated models of MeV-to-GeV-mass dark matter are those whose interactions with ordinary matter are mediated by new GeV-scale 'dark' force carriers (for example, a gauge boson that kinetically mixes with the SM photon) [5-8]. Such models readily account for the stability of dark matter and its predicted relic density, having important implications beyond the dark matter itself. In these scenarios, dark matter production at high-energy accelerators is generically buried under QCD background, making collider searches for light dark matter insensitive.

Dark matter in the $\mathrm{MeV}-\mathrm{GeV}$ range is therefore still relatively unexplored, and has been a topic of considerable recent interest (see e.g. [1] and references therein, and [912]). The sensitivity of past, ongoing, and proposed experiments to dark sector scenarios where dark matter interacts with the Standard Model through a kinetically mixed vector boson, $A^{\prime}$, is illustrated in Figure 1, originally from [1] (it should be noted that the sensitivity there reported for a "generic" JLAB experiment anticipated in [1] is somewhat 


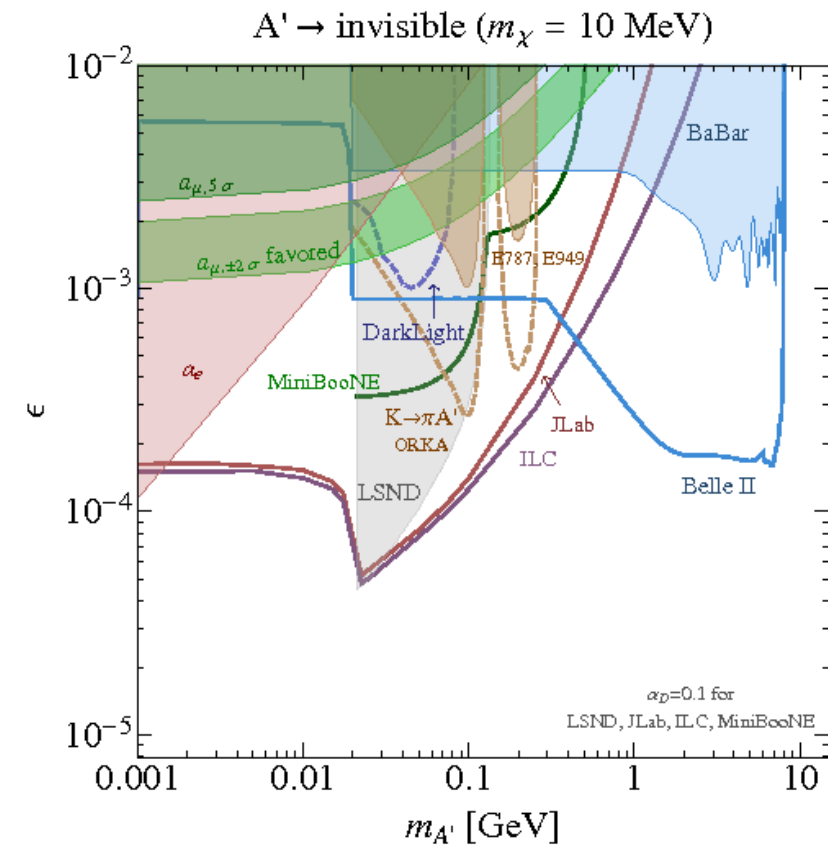

Figure 1: The international effort to search for GeV-scale dark matter and invisibly decaying vector mediators is partially summarized in the Report of the Community Summer Study 2013 (Snowmass) "New, Light, Weakly-Coupled Particles" subgroup [1], with additional references therein. In the above plot, representative sensitivity of several approaches is illustrated in the parameter space of mediator coupling $\epsilon$ vs. mediator mass $m_{A^{\prime}}$ for invisible decays of the mediator into dark matter candidates. The approaches include rare meson decay studies, proton and electron beam fixed target and B-factory collider searches. Shaded regions denote constraints from past experiments (LSND, muon and electron $g-2$, and $\mathrm{BaBar}$ ) and the region favored by the muon $g-2$ anomaly (lower green band); dashed lines correspond to potential sensitivities for proposed/future experiments. Importantly, the approaches shown cannot be compared modelindependently, and this plot is only representative of a particular parameter point in the kinetic mixing model of [1].

weaker, depending on $A^{\prime}$ mass, than the sensitivity of the BDX experiment here presented). While this figure illustrates the power and complementarity of the various experiments, it is of limited use for comparing experiments that use different search techniques, whose sensitivities change considerably for different parameter choices or model assumptions.

The considerable sensitivity of beam-dump experiments to light dark matter is underscored by the reach of running neutrino experiments [1, 9, 13-15]. For example, the LSND measurement of electron-neutrino scattering [16] can be used to derive the most stringent constraints to date on the parameter space for invisibly-decaying dark mediators that couple to both baryons and leptons [14]. That experiment delivered $\sim 10^{23} 800 \mathrm{MeV}$ protons to the LANSCE beam-dump. For very low mass $A^{\prime}$ s and dark matter sufficiently light $\left(100 \mathrm{MeV} \lesssim m_{A^{\prime}} \lesssim 2 m_{\chi}\right)$, the produced neutral pions have a small exotic decay into $A^{\prime}$ s which then decay to $\chi$. The $\chi$ can then scatter off electrons in the LSND detector via $A^{\prime}$-exchange. However, the sen- sitivity of LSND vanishes if the mediator couples only to leptons or baryons and is weakened if its coupling to either is suppressed. Relatedly, MiniBooNE is currently being used to search for light dark matter in a dedicated run of the BooNE proton beam at FNAL [17].

Recently it was shown that electron-beam fixed target experiments offer powerful sensitivity to a broad class of dark sector scenarios with particle dark matter in the $\mathrm{MeV}-\mathrm{GeV}$ mass range $[10,11,18]$. Electron beamdump experiments are complementary to dedicated efforts at proton beam facilities, have comparable DM scattering yield, can run parasitically and on a smaller scale than proton-beam counterparts, and benefit from negligible beam-related backgrounds. Such searches can dramatically improve sensitivity to $\mathrm{MeV}$-to-GeV mass dark matter and other long-lived weakly coupled particles, extending well beyond the reach of proposed neutrino-factory experiments and Belle-II projections. The power of electron beam dump experiments in this context is illustrated by the existing sensitivity of the SLAC E137 experiment [19]. That experiment was sensitive to invisibly decaying dark mediators produced in fixed target collisions involving 20 $\mathrm{GeV}$ electrons and the E137 beam-dump [20]. Despite the rather high energy threshold $(\sim 1 \mathrm{GeV})$ required to see secondary scattering of dark matter particles off electrons, and the small geometric acceptance, E137 has already investigated a region in the parameters space beyond that probed by proton beam-dumps, for intermediate masses.

\subsection{Dark-Photon and DM Interactions}

Whether the dark sector is quite simple or has a rich sector of light particles, the fixed-target phenomenology of stable $\chi$ s (or unstable $\chi$ s with lab-frame lifetimes $z \mu$ s) is usually well-described by the simplest case - a renormalizable $U(1)_{D}$ dark sector with a single stable matter particle $\chi$. For fermionic $\chi$ and $U(1)_{D}$ coupling to Standard Model matter via kinetic mixing,

$$
\begin{aligned}
\mathcal{L}_{\text {dark }} & =-\frac{1}{4} F_{\mu \nu}^{\prime} F^{\prime \mu \nu}+\frac{\epsilon_{Y}}{2} F_{\mu \nu}^{\prime} B_{\mu \nu}+\frac{m_{A^{\prime}}^{2}}{2} A_{\mu}^{\prime} A^{\prime \mu}+ \\
& +\bar{\chi}\left(i \not D-m_{\chi}\right) \chi,
\end{aligned}
$$

where $B_{\mu \nu}=B_{[\mu, v]}$ and $F_{\mu \nu}^{\prime}=A_{[\mu, v]}^{\prime}$ are respectively the hypercharge and dark-photon field strengths and $D_{\mu}=\partial_{\mu}+$ $i g_{D} A_{\mu}^{\prime}$ (and similarly for scalar $\chi$ ).

The kinetic mixing parameter $\epsilon_{Y}$ can arise generically from loops of heavy particles charged under both hypercharge and $U(1)_{D}$ and is naturally small, on the scale of $\frac{e g_{D}}{16 \pi^{2}} \log (M / \Lambda) \sim 10^{-5}-10^{-2}$, where $M$ is the mass of the particle in the loop and $\Lambda$ the theory cutoff scale. It is convenient to take as a free parameter $\epsilon \equiv \epsilon_{Y} \cos \theta_{W}$ (where $\theta_{W}$ is the weak mixing angle), rather than $\epsilon_{Y}$ itself.

Upon diagonalizing the kinetic mixing terms in (1), ordinary electrically charged matter acquires a "dark millicharge" coupling to the $A^{\prime}$ of strength $\epsilon e$, while the $\chi$ remains electrically neutral. As a consequence, long-lived dark sector particles $\chi$ couple to ordinary matter primarily through $A^{\prime}$ exchange. 
a)

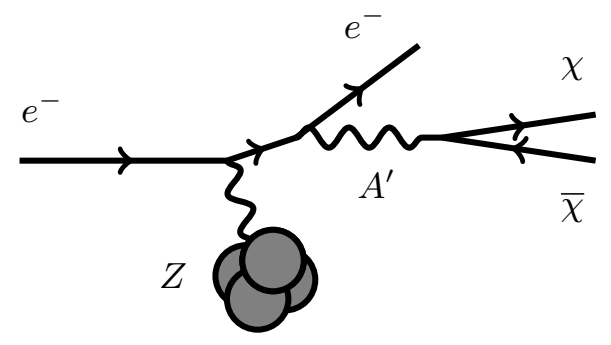

b)

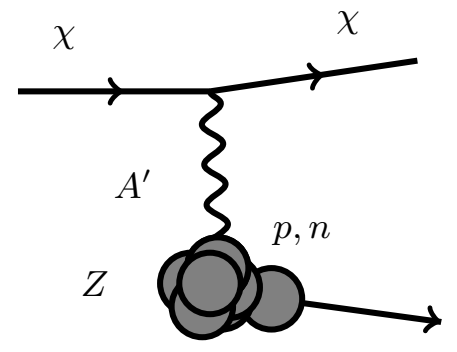

Figure 2: a) $\chi \bar{\chi}$ pair production in electron-nucleus collisions via the Cabibbo-Parisi radiative process (with $A^{\prime}$ on- or off-shell) and b) $\chi$ scattering off a detector nucleus and liberating a constituent nucleon. For the momentum transfers of interest, the incoming $\chi$ resolves the nuclear substructure, so the typical reaction is quasi-elastic and nucleons will be ejected.

In this theory, $\chi \mathrm{s}$ can therefore be pair-produced radiatively in electron-nucleus collisions in the dump (see Fig. 2a). A fraction of these relativistic particles then scatter off nucleons, nuclei, or electrons in the detector volume (see Fig. 2b).

If $m_{A^{\prime}}<2 m_{\chi}$, the dominant $\chi$ production mechanism in an electron fixed-target experiment is the radiative process illustrated in Fig. 2a) with off-shell $A^{\prime}$. In this regime, the $\chi$ production yield scales as $\sim \alpha_{D} \epsilon^{2} / m_{\chi}^{2}$ $\left(\alpha_{D} \equiv g_{D}^{2} / 4 \pi\right)$, while $\chi$-nucleon scattering in the detector via $A^{\prime}$ exchange (see Fig. 2b)) occurs with a rate proportional to $\alpha_{D} \epsilon^{2} / m_{A^{\prime}}^{2}$ over most of the mass range. Thus, the total signal yield scales as

$$
N_{\chi} \sim \frac{\alpha_{D}^{2} \epsilon^{4}}{m_{\chi}^{2} m_{A^{\prime}}^{2}} .
$$

If $m_{A^{\prime}}>2 m_{\chi}$, the secondary $\chi$-beam arises from radiative $A^{\prime}$ production followed by $A^{\prime} \rightarrow \bar{\chi} \chi$ decay. In this regime, the $\chi$ production and the detector scattering rates are respectively proportional to $\epsilon^{2} / m_{A^{\prime}}^{2}$ and $\alpha_{D} \epsilon^{2} / m_{A^{\prime}}^{2}$ and the signal yield scales as

$$
N_{\chi} \sim \frac{\alpha_{D} \epsilon^{4}}{m_{A^{\prime}}^{4}} .
$$

Thus, for each $\alpha_{D}$ and $m_{A^{\prime}}$, the $\epsilon$-sensitivity corresponding to a given scattering yield can be extracted. The characteristic momentum transfer in $\chi$-matter interactions is of order the $A^{\prime}$ mass. For $A^{\prime}$ masses from tens to hundreds of $\mathrm{MeV}, \chi$ can scatter coherently off a nucleus (with a $Z^{2}$-enhanced cross-section, but the lowest energy transfers and therefore highest radiological backgrounds)

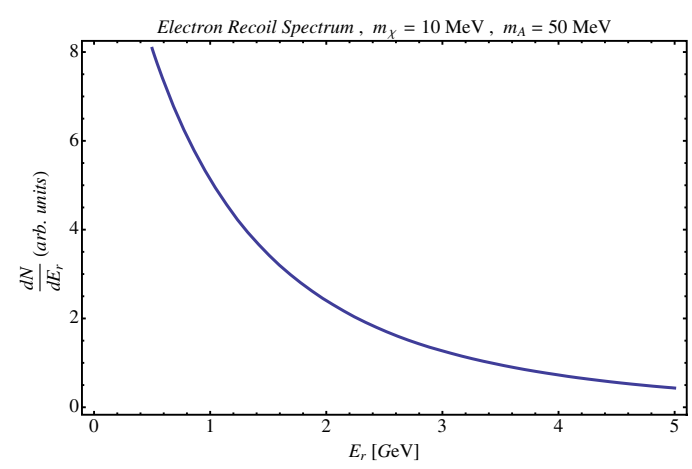

Figure 3: Energy distributions of scattered electrons for a specific combination of $\mathrm{M}_{\chi}$ and $\mathrm{M}_{A^{\prime}}$.

or eject a nucleon through a quasi-elastic scattering reaction $\chi p, n \rightarrow \chi p, n$, with characteristic recoil energy $E_{R} \sim m_{A^{\prime}}^{2} / 2 m_{N} \sim 1-50 \mathrm{MeV}$. Resonant single-pion and non-resonant double-pion production processes may also have appreciable yields, particularly for higher $A^{\prime}$ masses.

A second signal which is slightly sub-dominant but of considerable experimental importance is $\chi$-electron scattering. Because the electron is light and the $\chi$ are energetic, scattered electrons typically carry $\mathrm{GeV}$-scale energy and are therefore subject to much lower backgrounds than nucleon scattering. Figure 3 shows the lepton recoil energy for a specific choice of $\mathrm{M}_{\chi}$ and $\mathrm{M}_{A^{\prime}}$, considering a primary $11 \mathrm{GeV}$ electron beam. Indeed, for models with kinetically mixed mediators which produce both electronand nucleon-scattering signals, it is likely that electronrecoil searches at BDX will have the greatest sensitivity. At the same time, other models appear to have distinctive signals primarily in nucleon-scattering, for which BDX's unique sensitivity to nuclear recoils is ideal.

\section{The Beam Dump Experiment at Jefferson Laboratory}

\subsection{Experimental setup}

In the BDX experiment, a $\mathrm{O}\left(\mathrm{m}^{3}\right)$ calorimeter will be installed behind one of the Jefferson Laboratory highintensity experimental Halls (A and C). High energy (11 $\mathrm{GeV}$ ) electrons from the CEBAF accelerator will impinge on the beam-dump, possibly producing a secondary darkmatter beam. Dark matter particles will be detected trough the scattering on the detector material (electrons and quasifree nucleons), resulting in a visible energy deposition (see Figure 5 for a schematic).

This configuration is fully parasitic and does not affect the other operations foreseen in the experimental Hall. With beam currents up to $\simeq 100 \mu \mathrm{A}$, and considering an accelerator efficiency of $\simeq 50 \%$, the experiment would be capable to measure up to $10^{22}$ electrons on target per year, roughly 100 times the charge deposited on E137.

The main option considered so far in BDX is to construct a highly segmented, plastic-scintillator based detector. The choice of plastic scintillator was motivated by the necessity of employing a high density active target to 


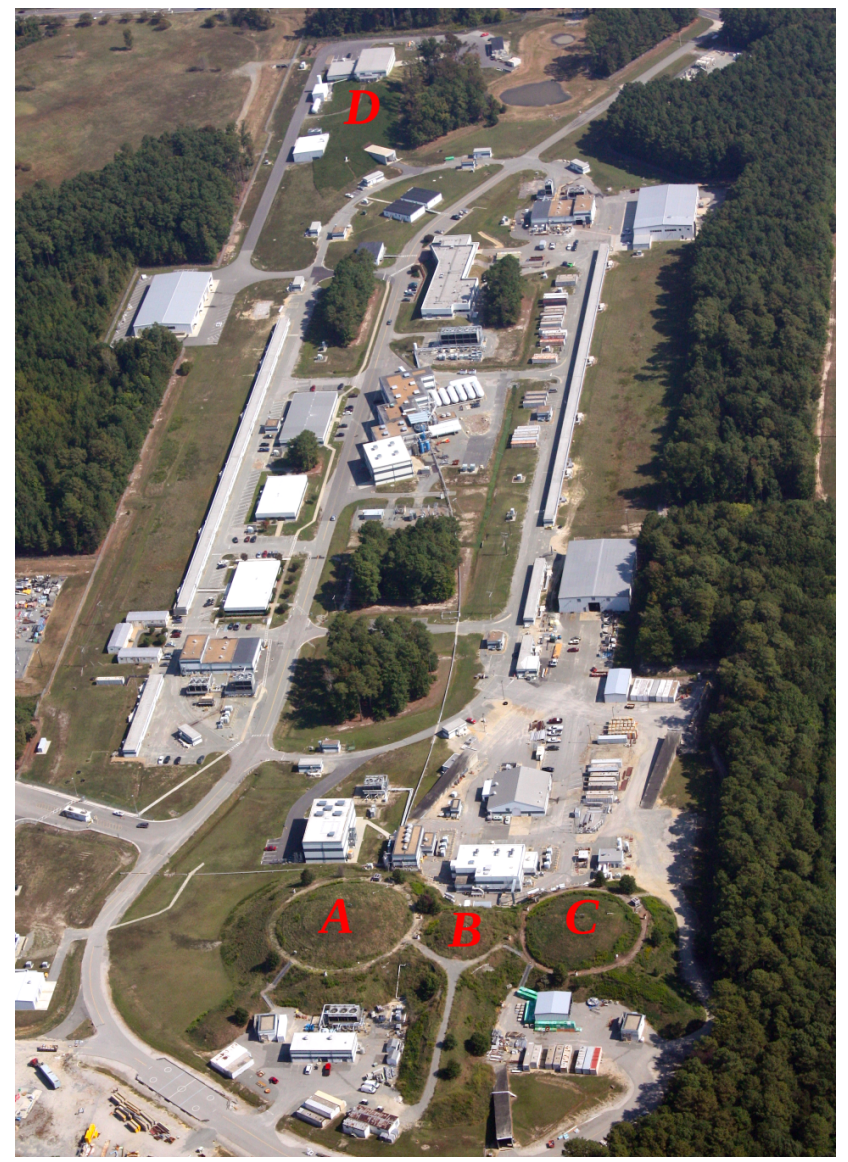

Figure 4: Aerial view of the CEBAF accelerator complex at Jefferson Laboratory, showing the race-track configuration of the accelerator. The four experimental Halls are highlighted. Figure taken from [21].

maximize the event yield. This also permits to build a large-volume detector with a versatile configuration, and reduced costs. To enhance the detector sensitivity to electromagnetic showers (induced by the $\chi \mathrm{e}^{-}$interaction), we plan to interleave plastic layers with lead-sheets, to increment the overall radiation length. Although plastic scintillator seems a promising solution, other choices are also currently being investigated, such as liquid scintillators and inorganic crystals.

The detector geometry is still being optimized, since it has to match the foreseen angular distribution of the secondary $\chi$ beam arising from the dump, that, in turns, depends on $m_{A}$ and $m_{\chi}$. However, in the kinematic range of interest $\left(m_{A}^{\prime} \lesssim 500 \mathrm{MeV}\right.$ and $m_{\chi} \lesssim 100 \mathrm{MeV}$ ), the primary electron beam energy is high enough to focus the secondary $\chi$ beam in the forward direction. A detector with a $50 \times 50 \mathrm{~cm}^{2}$ front-face placed $15 \mathrm{~m}$ downstream the beamdump has a $\simeq 95 \%(\simeq 60 \%)$ acceptance for $m_{A^{\prime}}=50 \mathrm{MeV}$, $m_{\chi}=10 \mathrm{MeV}\left(m_{A^{\prime}}=150 \mathrm{MeV}, m_{\chi}=68\right)$.

To exploit the forward $\chi$ kinematic, a possible choice is to construct a $2 \mathrm{~m}^{3}$ modular detector, with 20 $50 \times 50 \times 45 \mathrm{~cm}^{3}$ modules aligned along the primary beam axis to form a $9-\mathrm{m}$ long detector. Each module is made by a matrix of $3 \times 3$ independent optical channels, read at both ends by photomultiplier tubes. This configuration would have the advantage of permitting to probe different kinematic ranges by changing the modules alignment during data-taking. The low-mass region, corresponding to strongly-peaked forward $\chi \mathrm{s}$, would be explored by aligning all the modules along the beam direction, while the high-mass region, corresponding to a broader $\chi$ beam, would be better covered (due to the enhanced acceptance) by increasing the detector front-face, at the price of a reduced length.

Finally, to reduce the number of background hits, mainly due to cosmic muons and neutrons, the detector will be surrounded by an active veto, made by plastic scintillator counters, and by passive shielding (iron). The configuration we considered foresees a $1 \mathrm{~m}$ iron shield all around the detector, and assumes a 5\% inefficiency for the veto system.

\subsection{Experimental reach}

We evaluated the experimental reach by computing the foreseen number of background hits in the detector $N_{b c k}$ trough detailed Montecarlo simulations, and comparing this to the expected number of signal events $N_{\text {sig }}$ (as a function of the model parameters). The BDX experiment will be capable to observe a $\chi$ signal in the parameter space where $N_{s i g} \gtrsim(2 \div 3) \sqrt{N_{b c k}}$, i.e. where the expected number of signal events is higher than the background counts statistical fluctuations.

We performed a detailed calculation for the quasielastic nucleon channel, considering for two possible detection thresholds, $1 \mathrm{MeV}$ and $10 \mathrm{MeV}$ respectively. Results are summarized in Table 1, compared to the expected event yield for two benchmark scenarios: S.I $\left(m_{A}^{\prime}=50\right.$ $\mathrm{MeV}$ and $\left.m_{\chi}=10 \mathrm{MeV}\right)$ and S.II $\left(m_{A}^{\prime}=150 \mathrm{MeV}\right.$ and $m_{\chi}=68 \mathrm{MeV}$ ). Both scenarios are evaluated at $\alpha_{D}=0.1$ and $\varepsilon^{2}=1.5 \cdot 10^{-7}$. Given the much higher energy and the specific signal topology for the $\chi-e$ scattering (an electromagnetic shower developing along the primary $e^{-}$beam axis), we performed the reach calculation for this scenario assuming $N_{b c k}=0$. This assumption is somehow supported by the E137 experiment, that, imposing a $1 \mathrm{GeV}$ threshold, did not measure any hit during the 3-months run.

\begin{tabular}{|c|c|c|}
\hline & Counts $_{T h r=1 \mathrm{MeV}}$ & Counts $_{T h r=10 \mathrm{MeV}}$ \\
\hline \hline$\chi$ detection - S.I & $0.510^{6} \pm 700$ & $5.710^{4} \pm 240$ \\
\hline$\chi$ detection - S.II & $1.010^{4} \pm 100$ & $3.310^{3} \pm 60$ \\
\hline \hline Beam-rel bg & $100 \pm 10$ & $10 \pm 3$ \\
\hline \hline Beam-unrel bg & $1.610^{6} \pm 1300$ & $1.410^{6} \pm 1200$ \\
\hline
\end{tabular}

Table 1: Expected counts for 6 months run time at $100 \mu \mathrm{A}$ (corresponding to $10^{22}$ EOT) for signal, beam-related and beamunrelated backgrounds for $1 \mathrm{MeV}$ and $10 \mathrm{MeV}$ detection threshold.

Concerning the quasi-elastic nucleon channel, beamrelated backgrounds are mainly neutrons and neutrinos 

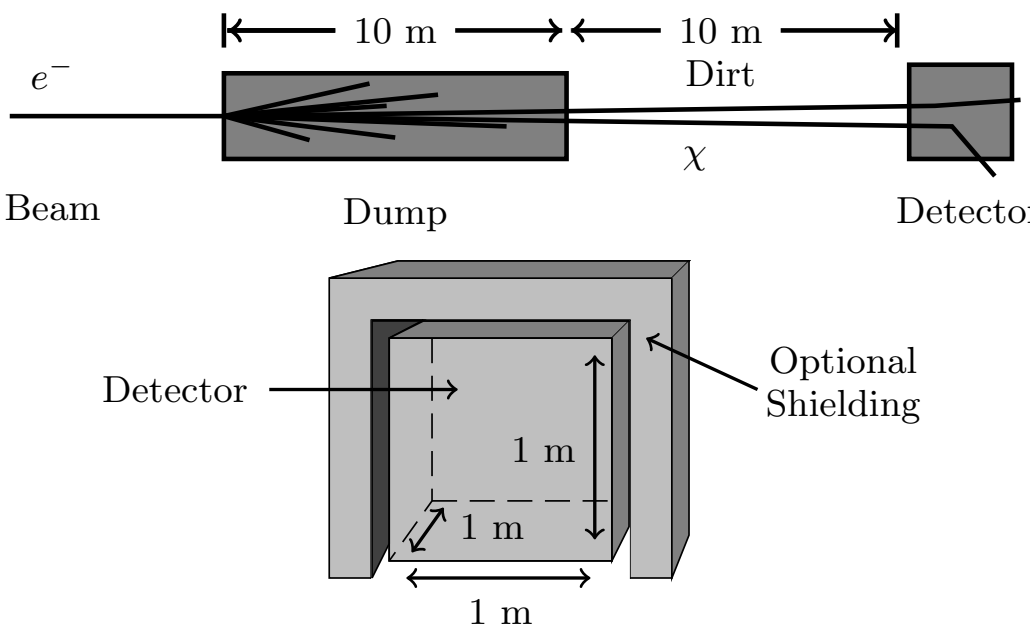

Figure 5: Schematic of the experimental setup. A high-intensity multi-GeV electron beam impinging on a beam-dump produces a secondary beam of dark sector states. In the foreseen setup, a $\mathrm{O}\left(\mathrm{m}^{3}\right)$ detector is placed downstream with respect to the beam-dump, surrounded by an active veto system and by passive shielding to reduce the number of hits due to cosmogenic backgrounds (muons and energetic neutrons).

produced in the dump that exit from it, scatter on the detector without triggering the veto, and release a visible amount of energy in a single optical channel, thus mimicking a $\chi-p$ event. Preliminary simulations [22] showed that $\mathrm{a} \simeq 1 \mathrm{~m}$ long dump, followed by $\simeq 8 \mathrm{~m}$ of iron shielding, is enough to reduce the neutrons contribution to a negligible count rate. The neutrino contribution, instead, can not be reduced by using passive shielding. Neutrinos are mainly produced from the at-rest decay of pions and muons in the dump, in the energy range $0-50 \mathrm{MeV}$. The incident flux on the detector, that scales linearly with the primary beam current, is $\simeq 2 \cdot 10^{7} \mathrm{~Hz} / \mu \mathrm{A}$, and the interaction cross section $\simeq 10^{-40} \mathrm{~cm}^{2}$, resulting in $\simeq 100$ counts in 1 year, for a beam current of $100 \mu \mathrm{A}$ and a detection threshold of 1 $\mathrm{MeV}$.

Beam-unrelated background is mainly due to cosmic neutrons, cosmic muons and their decay products, including rare decays of muons producing $\gamma \mathrm{s}$ between the passive shield and the active veto.

High-energy cosmic neutrons can mimic a $\chi-p$ interaction by producing a hit with visible energy deposition in a single detector optical channel. Considering the typical interaction length of neutrons in plastic, the thin $(\simeq 1-2$ $\mathrm{cm})$ active veto has a small chance to detect the incoming neutrons. On the other hand, the veto is sometimes useful to reject neutron events producing hadronic showers in the heavy passive shielding when some charged fragments reach the detector. The passive shield surrounding the detector, instead, introduces an effective energy cutoff in the primary energy spectrum, so that neutrons with energy lower than $50 \mathrm{MeV}(100 \mathrm{MeV})$ do not induce any visible hit in the detector, for an energy threshold of 50 $\mathrm{MeV}(100 \mathrm{MeV})$.

Cosmic muons are by far the main source of uncorrelated background in a plastic scintillator-based detector.
The most part of muons interacts with the veto counter and with the detector releasing the typical energy of a minimum ionizing particle, that can be tracked and rejected exploiting the detector segmentation. However, given the very high flux of primaries, even a small veto inefficiency results in a sizable contribution to the total background rate. Another sizable source of background is due to muons entering the detector volume and there stopping, releasing visible energy in a single hit. These particles only cross one side of the veto system, and, therefore, have an higher probability of not being properly identified. Furthermore, positive muons also induce a delayed hit due to the positron from the decay $\mu^{+} \rightarrow e^{+} \bar{\mu}_{\nu} \mu_{e}$. Negative muons, instead, are mainly captured by nuclei and do not result in a delayed hit.

Finally, the contribution of cosmic neutrinos is negligible, due to the low flux and the small interaction cross section.

Beam-uncorrelated background can be partially rejected by requiring a time coincidence between the accelerator RF signal and the event recorded by the detector. Even if the studied case of a plastic scintillator detector provides a sub-ns response to a $\chi$ scattering, the almostcontinuous structure of the CEBAF beam does not allow to take full advantage of this technique. In fact the bunch separation expected with the nominal CEBAF operations is $\Delta T=4 \mathrm{~ns}$, to be compared with the expected time resolution of $\sigma_{T} \simeq 100-200$ ps for $1 \mathrm{MeV}$ deposited energy. The background reduction factor, $R$, can be expressed as the ratio between the time coincidence window width $\left(3 \sigma_{T}\right)$ and $\Delta T$ providing:

$$
R=\frac{3 \sigma_{T}}{\Delta T} \sim 10-20 \%
$$

It is worth to notice that for the set up discussed in this paper, a detector placed $15 \mathrm{~m}$ away from the beam-dump, 


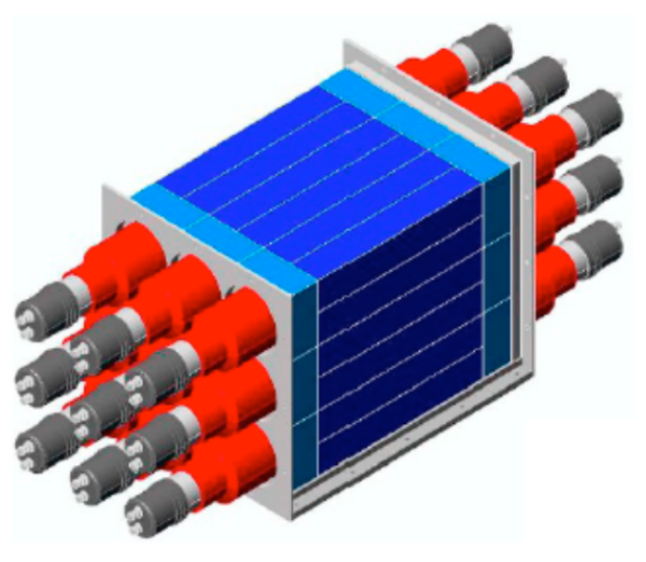

Figure 6: CAD representation of the CORMORINO detector, made by 9 bars of plastic scintillators read, each read on both sides by a a 3" PMTs.

$\chi$ masses between $0-500 \mathrm{MeV}$ result in a time-of-flight spread below $\sigma_{T}=200 \mathrm{ps}\left(0-170 \mathrm{MeV}\right.$ for $\left.\sigma_{T}=20 \mathrm{ps}\right)$. The same argument applies to the $\chi$ momentum spread: any $\mathrm{p}_{\chi}$ between $11 \mathrm{GeV}$ and $100 \mathrm{MeV}$ correspond to a time-of-flight spread below $\sigma_{T}=200 \mathrm{ps}(6 \mathrm{GeV}-350 \mathrm{MeV}$ for $\left.\sigma_{T}=20 \mathrm{ps}\right)$. This rational suggests that, for a fast detector $\left(\sigma_{T} \sim 70-100 \mathrm{ps}\right)$ the rejection factor for uncorrelated background can be reduced up to a factor of ten.

As previously discussed, the foreseen background contribution, and, therefore, the experiment sensitivity was evaluated trough detailed Montecarlo simulations. We plan to validate these by comparing background measurements and MC results from a well-defined and known experimental setup, employing an existing prototype of the full detector, CORMORINO. The CORMORINO detector is a $0.036 \mathrm{~m}^{3}$ cube made of plastic scintillator bars read by PMTs. The detector is made by nine optical channels, each consisting of four $5 \times 5 \times 30 \mathrm{~cm}^{3}$ plastic scintillator bars (NE102) individually wrapped in aluminised Mylar. The four bars are coupled on each side to a Photonis XP2312 3" photomultiplier via a $10 \times 10 \times 5 \mathrm{~cm}^{3}$ scintillator block, acting as light guide. Figure 6 shows a CAD drawing of the detector. We are currently building a hermetic chargedparticle veto counter based on $2 \mathrm{~cm}$ thick plastic scintillator planes to be placed in front of each of the 6 faces of the CORMORINO to reject cosmic muons and further reduce backgrounds.

We plan to perform a dedicated measurement campaign at INFN-LNS in Catania (Italy) to estimate the background contribution in a realistic experimental setup. The results will be scaled to the full detector and compared with Montecarlo simulations. Furthermore, during these measurements we will study the detector properties that directly affect the BDX performances, such as the detection thresholds and the time resolution.
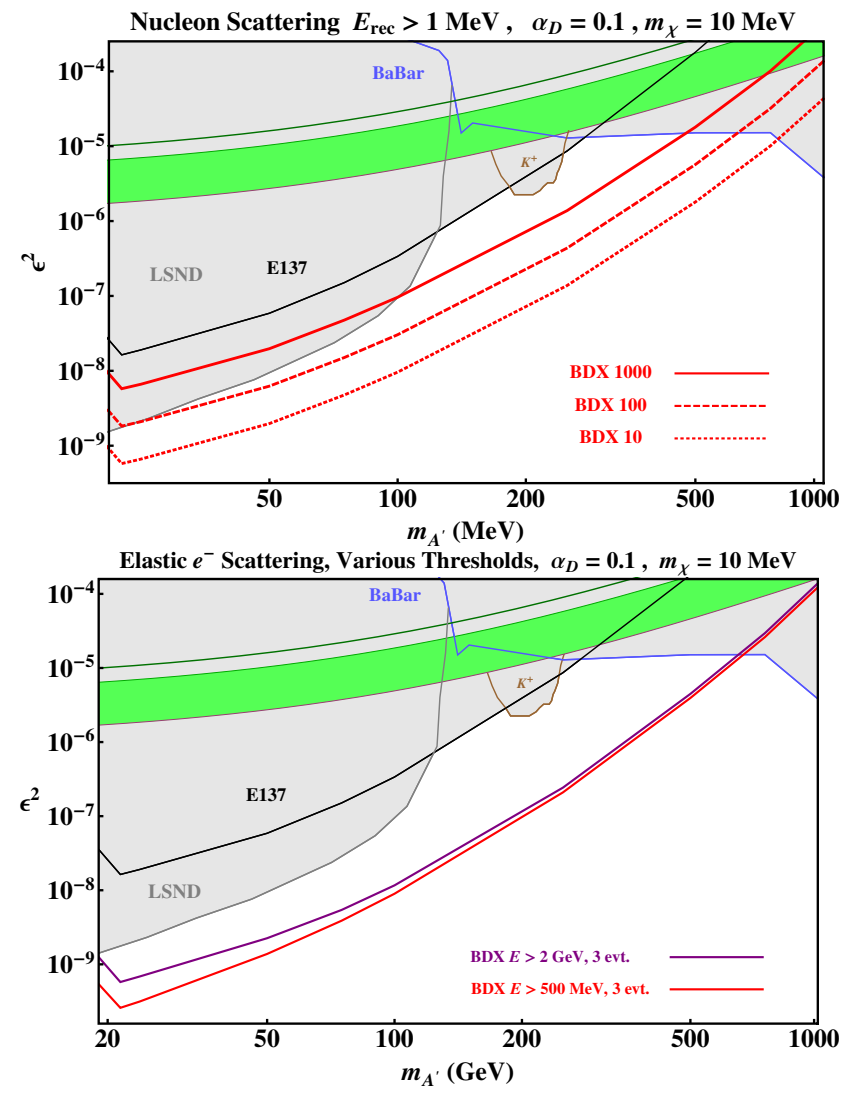

Figure 7: Top: Red curves show 10, 100, and 1000 event BDX yield projections for a kinetically-mixed dark-photon $\left(A^{\prime}\right)$ coupled to a nearly-invisible fermion $\chi$ in the quasi-elastic nucleon recoil channel with $10^{22}$ EOT. The dark photon is radiatively produced in electron-nucleus collisions in the beam-dump and decays promptly to yield $\bar{\chi} \chi$ pairs, which scatter off detector nucleons and deposit at least $1 \mathrm{MeV}$ of visible energy into the nuclear recoil signal. Bottom: BDX yield projections for a a kinetically-mixed dark-photon $\left(A^{\prime}\right)$ coupled to a nearly-invisible Dirac fermion $\chi$ in the electron recoil channel. The red and purple curves show 3 event yields with $500 \mathrm{MeV}$ and $2 \mathrm{GeV}$ cuts on electron recoils respectively.

\subsubsection{Results}

The BDX sensitivity to the kinetic mixing models previously discussed in the nucleon-recoil and electron channels are shown in Figure 16 and 17, respectively.

The exclusion regions shown in the plots were derived by constraints form cosmological observations, meson factories results and previous electron/hadron beamdump experiments. As explained before, limits imposed by E137 experiment on $\chi$-p elastic scattering were derived within a certain model from $\chi$-e elastic exclusion plot, being the experiment only able to detect multi-GeV electrons. BDX has the unique capability of being sensitive to different DM interaction mechanisms at the same time. The region potentially covered by JLab would therefore extend significantly the parameter space already excluded by previous experiments. 


\section{Conclusions}

The BDX experiment will search for dark-matter particles in the $\mathrm{MeV}-\mathrm{GeV}$ mass range, in an electron beam dump setup, probing a parameter space two orders of magnitude beyond the reach of existing and proposed experiments. Searches for particles in this mass range are motivated by models that feature a dark matter particle $\chi$ whose interactions with the Standard Model (SM) through a new massive dark photon generically appear with strength $\epsilon$ near $10^{-4}-10^{-2}$.

The experiment will measure dark matter particles produced in the beam dump of one of the high-intensity experimental halls at JLab and scattering on a plastic scintillator based detector placed $\simeq 15 \mathrm{~m}$ downstream. With a primary electron current up to $100 \mu \mathrm{A}$, and a 1 year run, BDX will be capable to collect up to $10^{22}$ EOT, approximately 2 orders of magnitude higher than previous electron beam-dump experiments.

BDX would detect the interaction of elastically scattered $\chi$ s off nucleons and electrons, by measuring the proton and electron recoil energies. The sensitivity of the beam dump experiment and the ability to reject backgrounds was studied based on a detailed Montecarlo simulation. These will be validated trough a dedicated measurement campaign at INFN-LNS using an existing detector prototype, CORMORINO.

In the absence of a signal and with energy thresholds as low as $1 \mathrm{MeV}$ for detecting $\chi-p$ interactions, the experiment would be able to set limits on the production of dark matter with masses in the range between 100 and 500 $\mathrm{MeV}$ and coupling constants $\epsilon^{2}$ between $10^{-7}$ and $10^{-5}$, dominated by statistical uncertainty of cosmogenic backgrounds. Elastic $\chi-e$ interactions with thresholds between 0.5 and $2 \mathrm{GeV}$ and essentially no background can be used to set limits on the production of dark matter with masses in the range between 20 and $700 \mathrm{MeV}$ and coupling constants $\epsilon^{2}$ between $10^{-9}$ and $10^{-5}$. These regions of masses and coupling strengths exceed the expected sensitivity of previous, existing, and proposed experiments by over two orders of magnitude.

\section{References}

[1] R. Essig et al., arXiv:1311.0029 [hep-ph].

[2] R. Essig et al., Phys. Rev. Lett. 109, 021301 (2012)

[3] R. Essig, J. Mardon and T. Volansky, Phys. Rev. D 85, 076007 (2012)

[4] P. W. Graham et al., Phys. Dark Univ. 1, 32 (2012)

[5] C. Boehm, T. A. Ensslin and J. Silk, J. Phys. G 30, 279 (2004)

[6] C. Boehm and P. Fayet, Nucl. Phys. B 683, 219 (2004)

[7] M. Pospelov, A. Ritz and M. B. Voloshin, Phys. Lett. B 662, 53 (2008)

[8] N. Arkani-Hamed et al., Phys. Rev. D 79, 015014 (2009)

[9] R. Dharmapalan et al. [MiniBooNE Collaboration], [arXiv:1211.2258 [hep-ex]]

[10] M. D. Diamond and P. Schuster, Phys. Rev. Lett. 111, 221803 (2013)

[11] E. Izaguirre et al., [arXiv:1403.6826 [hep-ph]]

[12] D. E. Morrissey and A. P. Spray, [arXiv:1402.4817 [hep-ph]]

[13] B. Batell, M. Pospelov and A. Ritz, Phys. Rev. D 80, 095024 (2009)

[14] P. deNiverville, M. Pospelov and A. Ritz, Phys. Rev. D 84, 075020 (2011)

[15] P. deNiverville, D. McKeen and A. Ritz, Phys. Rev. D 86, 035022 (2012)

[16] L. B. Auerbach et al. [LSND Collaboration], Phys. Rev. D 63, 112001 (2001)

[17] R. Dharmapalan et al. [MiniBooNE Collaboration], Proposal to the FNAL PAC, P - 1032, Dec 2013

[18] E. Izaguirre et al., Phys. Rev. D 88, 114015 (2013)

[19] J. D. Bjorken et al., Phys. Rev. D 38, 3375 (1988).

[20] B. Batell, R. Essig and Z. Surujon, Phys. Rev. Lett. 113, no. 17, 171802 (2014)

[21] www.jlab.org/photos

[22] M. Battaglieri et al. (BDX collaboration). [arXiv:1406.3028 [physics.ins-det]] 